\title{
3D Gaze Point Localization and Visualization Using LiDAR-based 3D Reconstructions
}

\author{
James Pieszala* \\ Gabriel Diaz \\ Jeff Pelz \\ Rochester Institute of Technology Rochester Institute of Technology Rochester Institute of Technology \\ Jacqueline Speir \\ West Virginia University \\ Reynold Bailey \\ Rochester Institute of Technology
}
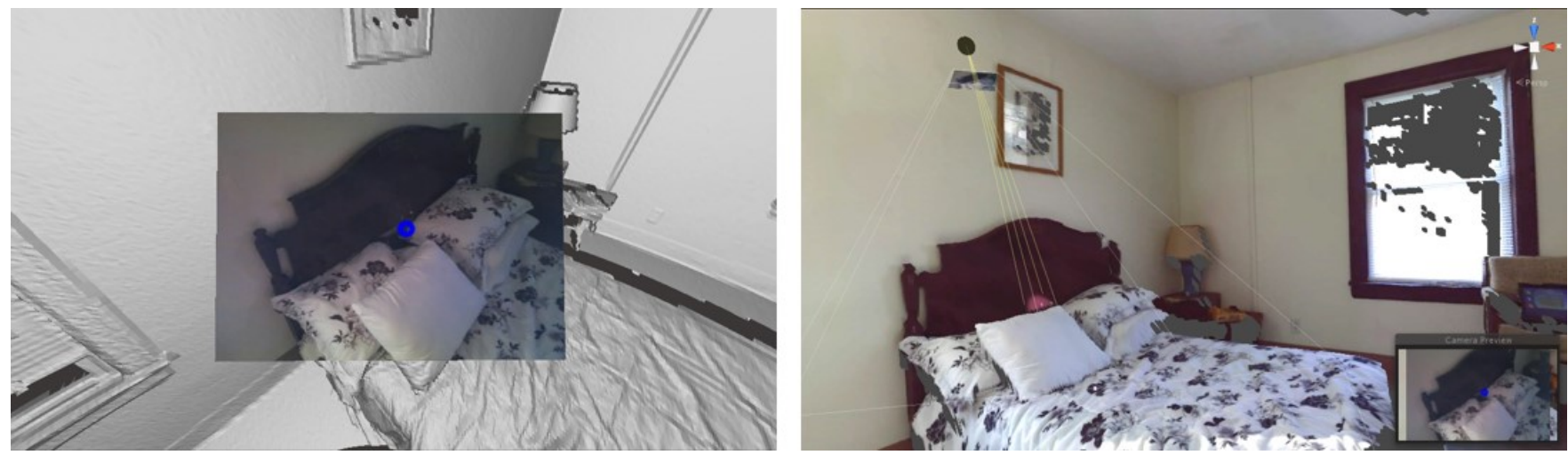

Figure 1: Alignment of single frame from a head mounted eye-tracker with $3 D$ reconstructed scene. (Left) First-person perspective - the blue marker indicates the current fixation point and the $3 D$ model is shown without RGB texturing. (Right) Third-person perspective - showing eye-tracker scene camera pose and corresponding 3D point-of-regard, 3D model is depicted with high resolution LiDAR texturing.

\section{Abstract}

We present a novel pipeline for localizing a free roaming eye tracker within a LiDAR-based 3D reconstructed scene with high levels of accuracy. By utilizing a combination of reconstruction algorithms that leverage the strengths of global versus local capture methods and user-assisted refinement, we reduce drift errors associated with Dense-SLAM techniques. Our framework supports regionof-interest (ROI) annotation and gaze statistics generation and the ability to visualize gaze in $3 \mathrm{D}$ from an immersive first person or third person perspective. This approach gives unique insights into viewers' problem solving and search task strategies and has high applicability in complex static environments such as crime scenes.

Keywords: 3D reconstruction, eye-tracking, 3D point-of-regard, computer vision, virtual reality

Concepts: •Computing methodologies $\rightarrow$ Computer vision; Tracking; Reconstruction; Virtual reality;

*e-mail: jkp3330@rit.edu

Permission to make digital or hard copies of all or part of this work for personal or classroom use is granted without fee provided that copies are not made or distributed for profit or commercial advantage and that copies bear this notice and the full citation on the first page. Copyrights for components of this work owned by others than ACM must be honored. Abstracting with credit is permitted. To copy otherwise, or republish, to post on servers or to redistribute to lists, requires prior specific permission and/or a fee. Request permissions from permissions@acm.org. (c) 2016 ACM.

ETRA 2016, March 14 - 17, 2016, Charleston, SC, USA

ISBN: 978-1-4503-4125-7/16/03

DOI: http://dx.doi.org/10.1145/2857491.2857545

\section{Introduction and Background}

The field of 3D scanning and geometric reconstruction has been changing at an unprecedented rate over the past 20 years. The commercialization of competing 3D data acquisition technologies has served to lower prices and empower practitioners in many disciplines. For example, automated scanning techniques are increasingly being used to document crime scenes. These scans are valuable to forensic scientists as they can be revisited as many times as desired, and by as many experts as needed, over the course of an investigation.

The ability to localize a head-mounted eye-tracker and visualize the $3 \mathrm{D}$ point-of-regard within reconstructed environments provides valuable insights into viewers' problem solving and spatial processing strategies. However, existing visualization techniques, developed for remote eye-tracking systems, are not directly applicable with head mounted eye-trackers. In remote systems, the relationship between the eye-tracker and display on which the stimuli are presented is fixed, making it straightforward to visualize the gaze behavior of multiple subjects. On the other hand, with head mounted eye-trackers, the viewer can move freely about the $3 \mathrm{D}$ environment, hence both the perspective and stimuli will differ across subjects. Early approaches to address this challenge relied on the creation of a panorama using key frames from one subject's eyetracker scene camera video [Pelz et al. 2011]. The gaze points gathered from all subjects performing the task were then mapped onto this panorama. However such approaches require that viewers observe the scene from a similar vantage point and do not preserve the relative positions of objects in the scene.

More recently, with the advances in 3D scanning, reconstruction technologies, and computer vision algorithms, more robust techniques for 3D gaze analysis are beginning to emerge [Paletta et al. 2013; Lidegaard et al. 2014; Booth et al. 2014]. However, techniques for localization of the head and eye-tracker within the $3 \mathrm{D}$ 


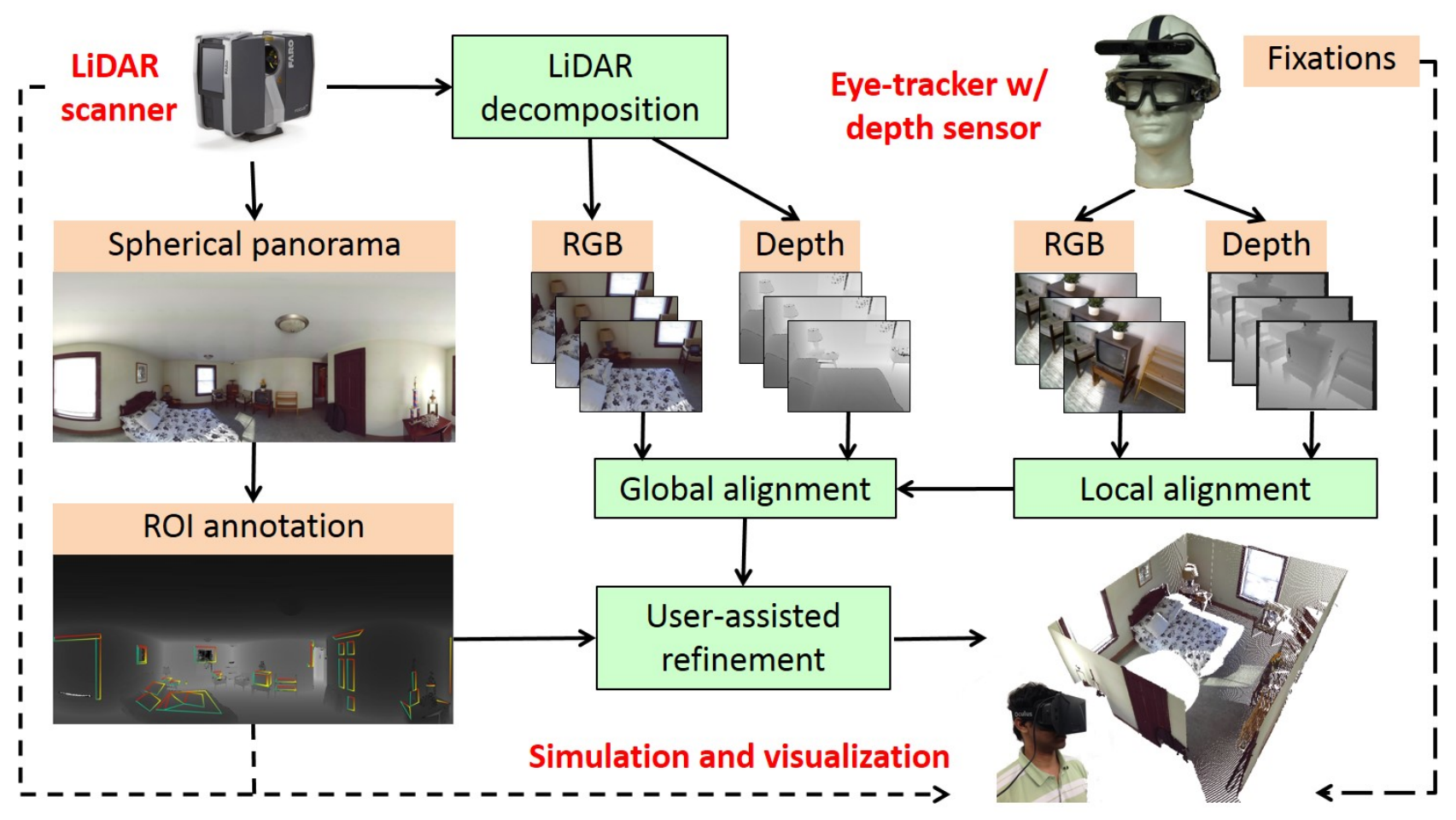

Figure 2: System overview and data flow.

scene often still rely on external motion capture cameras [Essig et al. 2012] which require considerable setup and calibration time thus making them impractical for many applications.

The pipeline described in this paper enables the visualization of gaze data from multiple viewers on a 3D reconstructed scene without the need for external motion capture. Subjects are free to move about the scene which leads to more natural task performance. Furthermore, since it is not necessary to warp the scene camera video into a flat panorama, our system preserves the relative positions of the objects in the scene during the visualization process.

Our pipeline utilizes a combination of global and local 2D and 3D registration techniques to align the imagery from a head-mounted eye-tracker and a head-mounted depth camera with depth and RGB data from a high resolution LiDAR scanner. Our pipeline supports region-of-interest (ROI) annotation which facilitates the generation of ROI-based gaze statistics. The user-created ROI annotations are also leveraged to further refine the alignment between the eye-tracker scene-camera imagery and the LiDAR 3D data. Figure 1 shows two perspectives of one frame of this alignment - a first-person view and a third-person view. This approach solves the problem of maintaining the 3D scene context and grants researchers increased insight into subjects' problem solving strategies. Visualizations can be created which highlight the regions of the scene that were attended to (or not attended to) by the subjects.

The finalized camera odometry, ROI annotations, eye-tracker event data, and relevant 3D models are sent to a simulation and visualization application. This application constructs virtual camera models corresponding to the recovered camera parameters and projects $2 \mathrm{D}$ gaze events onto the 3D scene model. The application is built using the Unity3D framework, and allows for a variety of interactive options including first-person and third-person playback perspectives, 3D heat map generation, active ROI visualization, and 3D gaze statistics generation. Using the registered RGB data from a
FARO LiDAR scanner, we can easily apply high resolution texturing to the scene as shown in Figure 1 (Right). For real-time interaction and rendering, the high resolution textures can be applied to decimated meshes and still maintain visual detail. Our visualization system is fully operable as a VR solution with Oculus Rift for immersive first-person or third-person simulations. Figure 6 (Top) shows a stereoscopic VR view of a 3D heat map.

\section{System Design}

Our goal is to incorporate recent advances in Dense-SLAM based $3 \mathrm{D}$ reconstruction with the global accuracy of LiDAR point clouds to facilitate the localization of head mounted eye trackers and associated 3D points-of-regard. To accomplish this, we seek to balance the disadvantages of some stand-alone solutions with the advantages of others. For instance, low-cost RGB-D capture devices such as the Microsoft Kinect provide ease of use and mobility but often suffer from noisy alignments and global drifting; on the other hand, LiDAR based scanners produce highly accurate global 3D point clouds but are often restricted to rigid anchor points. Recent innovations in Dense-SLAM based reconstructions [Xiao et al. 2013; Whelan et al. 2012; Choi et al. 2015] have mitigated drift and erroneous alignments to varying degrees. Our strategy proceeds by leveraging these existing solutions and anchoring a roaming eyetracker and depth sensor with LiDAR tie points. We take advantage of user-created annotations to further refine the alignment between the eye-tracker scene camera imagery and the LiDAR 3D data. This approach produces high accuracy camera odometries with correspondingly high quality $3 \mathrm{D}$ models that can be used in the research of 3D gaze behavior. Our approach is specifically designed for indoor static scenes with search tasks requiring deliberate examination of objects; which, typically involve viewing distances within the range of the roaming depth sensor. Figure 2 provides an overview of our system and illustrates the general flow of information through our pipeline. 


\subsection{Equipment and Data Collection}

The data used in this paper to demonstrate the capabilities of our pipeline were collected at a crime scene training facility. The data is comprised of 39 registered LiDAR scans collected using a FARO Focus3D laser scanner. A typical indoor scan consists of 10.8 million data points. In addition, each scan also captures a high resolution spherical panorama which we later use for global alignment, texture mapping and ROI annotation.

For eye-tracking, we used a SensoMotoric Instruments Eye Tracking Glasses (ETG) with a 1280 x 960 scene camera operating at $24 \mathrm{fps}$ and $640 \times 480$ binocular eye-cameras operating at $60 \mathrm{fps}$. A PrimeSense Carmine depth sensor was fixed to the head of the viewer in close proximity to the SMI eye-tracker. The PrimeSense sensor is equipped with 640 x 480 RGB and depth cameras operating at $30 \mathrm{fps}$. While not an elegant solution, this approach facilitates higher accuracy alignments and the depth information from the PrimeSense sensor can also be used to fill gaps in the LiDAR data. Future head-mounted eye-tracking solutions with integrated depth sensors would be a more practical approach. Members of our research team took turns walking through the crime scene facility using this system to collect pilot data.

\subsection{Local Alignment}

Local alignment refers to the serial alignment of consecutive camera frames from the same device. Local alignment is performed on the RGB video feed from the head-mounted depth sensor. Each frame is first processed using a wavelet based blur detector. If a frame exhibits significant blur due to rapid head movement, it is ignored and flagged as a break in the continuous alignment. In the event that an entire fixation is lost due to blurry frames, the least blurry flagged frame can be reintroduced and aligned with manual input during the final refinement stage. We have found this approach to be reasonable for relatively deliberate tasks, such as crime scene examinations.

Once the camera frames have been grouped into continuous tracks, each group is fed into a Dense-SLAM based alignment algorithm. Our approach is adapted from Xiao et al. [2013]. We use their SIFT feature-based serial alignment technique where corresponding depth coordinates are fed into a RANSAC 3-point-algorithm which solves for relative camera pose. Additional fine tuning is done with ICP and generalized bundle adjustment. In our implementation, we omit their loop closure module since the corresponding LiDAR associations make it redundant. The reason for performing these local alignments is that if a subsequent LiDAR alignment fails, the relative local associations will have already been computed and maintained.

\subsection{Global Alignment}

Global alignment refers to aligning the frames from the roaming devices (eye-tracker and depth camera) with the LiDAR 3D data. First we process the LiDAR scans to produce RGB-D frames. Since the LiDAR scanner uses spherical data acquisition, we project the RGB and depth data onto virtual planar frames, similar to Zhang et al. [2014]. This technique is easily extended to any number of registered LiDAR clouds. Following a similar process as the local alignment procedure described above, we perform brute force matching of each decomposed LiDAR frame against each roaming frame using a modified version of Xiao et al.'s algorithm [2013]. Frames that have not been globally registered are adjusted relative to their closest globally aligned neighboring frame. If necessary, additional bundle adjustment can be done to further reduce alignment errors. As a final step the offset between the eye-tracker and head-mounted
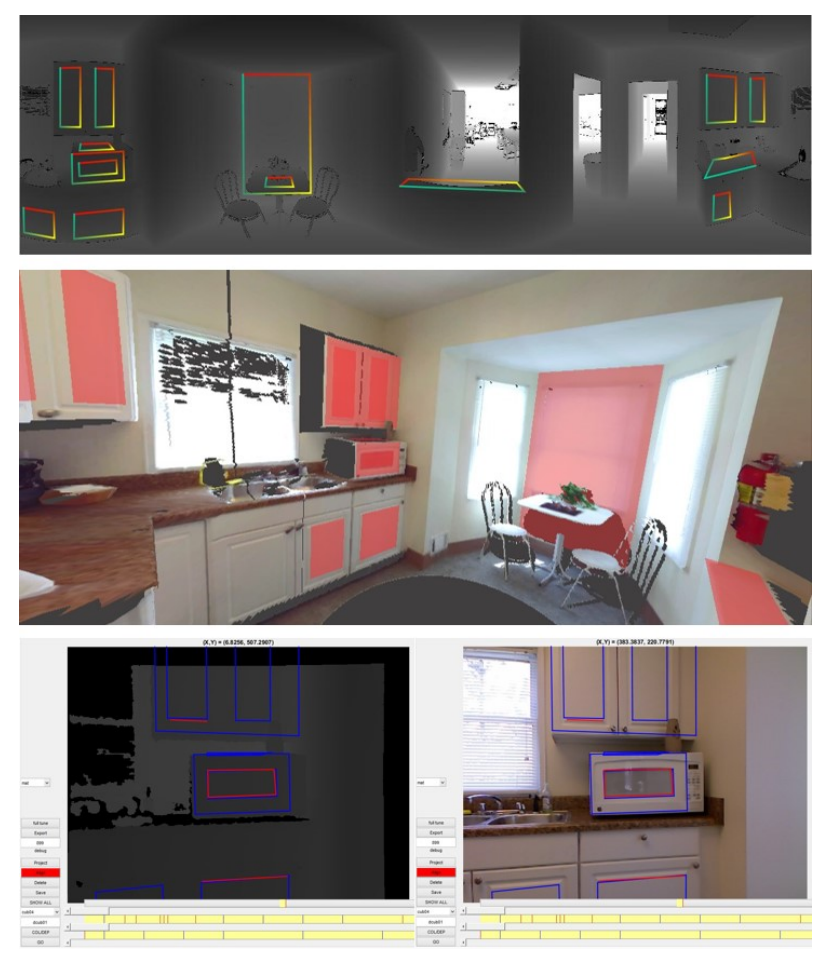

Figure 3: ROI Annotations. (Top) User-generated ROIs visualized on LiDAR spherical depth panorama. (Middle) Same annotations depicted on $3 D$ visualization. (Bottom) User interface for alignment verification and misaligned frame correction. The blue regions represent the projected ROIs. The red regions show the manually adjusted ROIs.

depth sensor is computed using a general perspective-n-point algorithm.

\subsection{ROI Annotations}

Typically in eye-tracking experiments, task-relevant objects or regions of interest (ROIs) are identified and annotated. These ROIs can then be used in the reporting of gaze statistics. In our pipeline, the user-created ROIs are also utilized to verify the accuracy of the recovered scene-camera odometry. This is accomplished by projecting the ROI's corresponding $3 \mathrm{D}$ points onto the localized camera frames. Once this is done, misaligned regions are easily noticed in our user interface and can be manually adjusted as shown in Figure 3. Any adjustment results in a camera matrix correction that is propagated to neighboring frames.

\section{Results \& Discussion}

As expected, camera tracks at slower speeds exhibited the highest automated local alignment accuracy due to less motion blur. Currently our wavelet-based blur detector uses a manually set conservative threshold to flag blurry frames. Using this approach across all of our data sets we observe a worst-case of $36 \%$ of frames being flagged. Nonetheless, the subsequent global alignment and user refinement still produce acceptable results. For example, Figure 4 illustrates the locally aligned camera paths from the head-mounted depth sensor in different colors. Yet, the global alignment stage is able to correlate these disparate groups. The occurrence of blurry frames drops off significantly when the viewer is engaged in deliberate visual scanning of the scene or if we only consider frames 


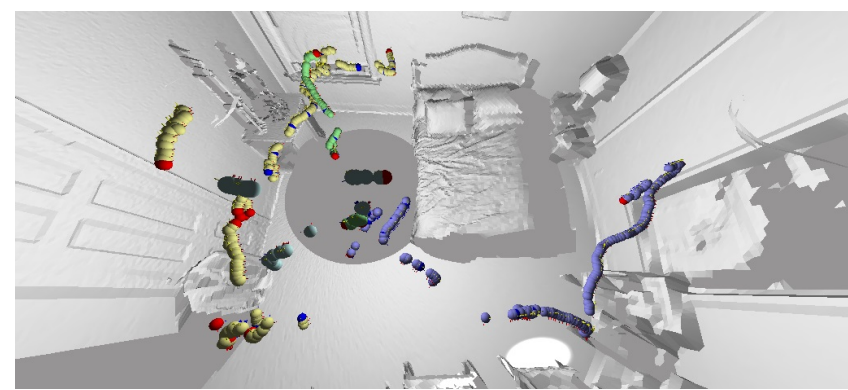

Figure 4: Locally-aligned camera path groups from the headmounted depth sensor shown in different colors.

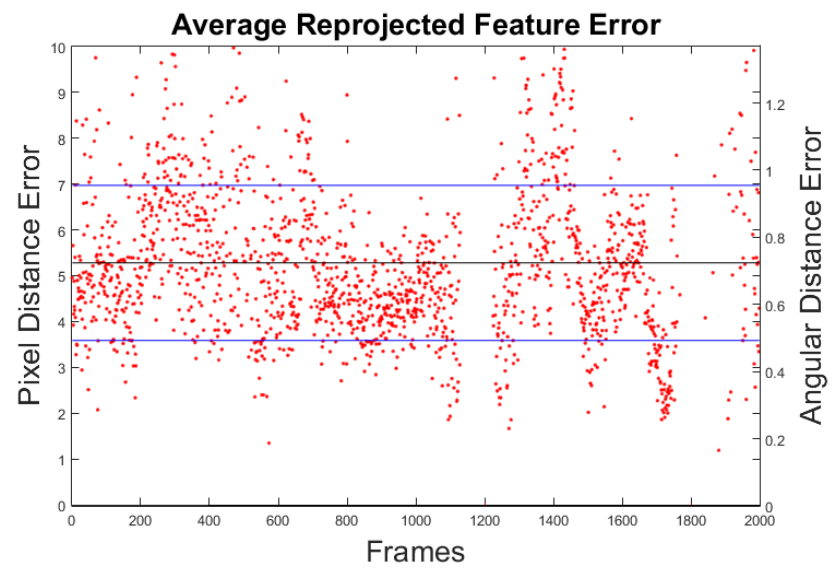

Figure 5: Alignment accuracy per frame. Black horizontal line indicates the mean and blue horizontal lines indicate the standard deviation for the dataset.

in which fixations occur (as is typically done in eye-tracking research). We envision using more elaborate threshold optimization techniques and higher-speed cameras to further improve local alignment percentages.

Figure 5 shows frame alignment accuracy for a typical dataset. The error metric is computed using 2D pixel distances between matched features used in the global alignment stage. Average recovered gaze position error is 0.5 angular degrees with a standard deviation of 0.15 degrees.

\section{Conclusion \& Future Work}

By augmenting Dense-SLAM based reconstruction techniques with the global accuracy of LiDAR clouds, our results show a feasible solution to eye-tracker localization and accurate 3D point-of-regard recovery; with optimal applicability for deliberate examination of indoor static scenes. There are a number of avenues for future work. For example, to further increase alignment accuracy and automation, more robust camera calibration is essential [Khoshelham and Elberink 2012] and consecutive roaming depth frames can be denoised as done in [Xiao et al. 2013]. We are presently working on a solution to automatically use data from the head-mounted depth sensor to fill gaps in the LiDAR data. This will be invaluable for additional ROI annotation, complete 3D point-of-regard statistical analysis, and better visualization coverage.

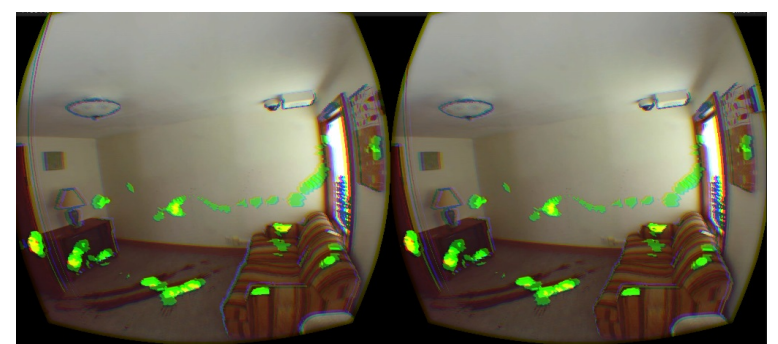

Figure 6: Heat map visualization in stereoscopic VR.

\section{Acknowledgements}

This material is based on work supported be the National Science Foundation under Award No. IIS-0952631. Any opinions, findings, and conclusions or recommendations expressed in this material are those of the author(s) and do not necessarily reflect the views of the National Science Foundation.

\section{References}

Booth, T., Sridharan, S., Bethamcherla, V., AND BaILEY, R. 2014. Gaze3D: Framework for gaze analysis on 3D reconstructed scenes. In Proceedings of ACM Symposium on Applied Perception, ACM, New York, USA, SAP '14, 67-70.

Choi, S., Zhou, Q.-Y., AND Koltun, V. 2015. Robust reconstruction of indoor scenes. In Proceedings of the IEEE Conference on Computer Vision and Pattern Recognition, 5556-5565.

Essig, K., Dornbusch, D., Prinzhorn, D., Ritter, H., MAYCOCK, J., AND SCHACK, T. 2012. Automatic analysis of 3D gaze coordinates on scene objects using data from eyetracking and motion-capture systems. In Proceedings of Symposium on Eye Tracking Research and Applications, ACM, 37-44.

Khoshelham, K., And Elberink, S. O. 2012. Accuracy and resolution of kinect depth data for indoor mapping applications. Sensors 12, 2, 1437-1454.

LidegaArd, M., Hansen, D. W., And Krüger, N. 2014. Head mounted device for point-of-gaze estimation in three dimensions. In Proceedings of the Symposium on Eye Tracking Research and Applications, ACM, New York, NY, USA, ETRA '14, 83-86.

Paletta, L., Santner, K., Fritz, G., Hofmann, A., LoDRON, G., Thallinger, G., AND MAYER, H. 2013. FACTSa computer vision system for 3D recovery and semantic mapping of human factors. In Computer Vision Systems. Springer, 62-72.

Pelz, J., Kinsman, T., And Evans, K. 2011. Analyzing complex gaze behavior in the natural world. SPIE-IS\&T Human Vision and Electronic Imaging XVI, 1-11.

Whelan, T., Kaess, M., Fallon, M., Johannsson, H., LEONARD, J., AND MCDonald, J. 2012. Kintinuous: Spatially extended kinectfusion.

Xiao, J., Owens, A., And Torralba, A. 2013. SUN3D: A database of big spaces reconstructed using SFM and object labels. In Computer Vision (ICCV), 2013 IEEE International Conference on, IEEE, 1625-1632.

Zhang, Y., Song, S., Tan, P., And XiaO, J. 2014. Panocontext: A whole-room 3D context model for panoramic scene understanding. In Computer Vis. ECCV 2014. Springer, 668-686. 